Supporting information for

\title{
Photoactivation of the Photoactive Yellow Protein: Why photon absorption triggers a trans-to-cis isomerization of the chromophore in the Protein
}

\author{
Gerrit Groenhof, Mathieu Bouxin-Cademartory, Berk Hess, Sam de Visser, Herman J.C. \\ Berendsen, Massimo Olivucci, Alan E. Mark, Michael A. Robb \\ Department of biophysical chemistry, University of Groningen, Nijenborg 4, 9747 AG Groningen, The \\ Netherlands \\ Department of theoretical chemistry, King's College London, Strand London WC2R 2LS, United \\ Kingdom \\ Dipartimento di Chimica, Università di Siena, Via Aldo Moro, I-53100 Siena, Italy
}

\section{Proton transfer step}

After the photoisomerization the PYP enters the third stage of the photocycle. In this stage the chromophore becomes protonated and the protein unfolds. The timescales of these processes are in the order of microseconds. As we were able to simulate only a few nanoseconds, reaching the unfolded conformation is not the purpose of continuing the simulations after the isomerization. Rather, the aim was to find short term effects of the isomerization. As was mentioned in the text, the isomerization did not cause any destabilization in the protein in the few nanoseconds simulated. But the affinity of the chromophore to take up the proton from the glutamic acid (Glu46) increased, and upon transfer of that proton, the protein became unstable.

Figure 5 of the paper shows the energy difference before and after proton transfer during 2 nanoseconds of simulation. However, in this analysis the proton was transferred instantaneously from the Glu46 sidechain to the chromophore. That is, in a frame in which the Glu46 sidechain is protonated, the proton was simply removed from that sidechain and added to the phenolate oxygen atom of the chromophore and the total energy of the system was re-evaluated. The assumption behind this approach is that the proton behaves adiabatically. The proton is much lighter than the other atoms in the system (mainly carbon, nitrogen, oxygen and sulphur), and therefore moves many times faster. In the adiabatic approach, the proton experiences a static field of the other atoms. Its motion only depends on the configuration of the heavy atoms, which can be considered frozen. This approach however is only truly valid if the transfer is dominated by tunneling through that barrier, (an instantaneous process). Tunneling is most efficient if the barrier is narrow and the reactant (deprotonated chromophore and protonated glutamic acid sidechain) and product state (protonated chromophore and deprotonated glutamic acid sidechain) have equal energies.

However, if the barrier for transfer is high and wide, the adiabatic approximation is not valid. Instead, the reorganization of the other atoms should be taken into account. The proton can not be transferred instantly. While the proton is moving from the donor (Glu46) to the acceptor (the chromophore) all atoms of the environment, consisting of the rest of the protein, the water molecules and the $\mathrm{Na}^{+}$ions, adjust their positions too. The conformational relaxation of the environment contributes to the overall energy that is required for transfering the proton. In fact, there must be a substantial entropic contribution to the free energy due to the reorientation and that will counterbalance much, if not most, of the energy.

To estimate the magnitude of the re-organization energy, two frames were taken, one from a simulation with an isomerized chromophore $(\mathrm{pR})$ and one from a simulation with an unisomerized chromophore $(\mathrm{pG})$. In each frame the protonation states in the chromphore pocket were changed from a protonated glutamic acid sidechain and a deprotonated chromophore sidechain to a protonated chromophore and a deprotonated glutamic acid sidechain and the resulting conformation saved. Each of these four frames was then rigorously optimized. First the energy of the total system was minimized using a first steepest descent approach then using a conjugate gradient algorithm. During these QM/MM computations, the QM part consisted of the chromophore and the sidechains of Glu46 and Tyr42, which were modeled with the semi-empirical PM3 Hamiltonian (see methods). After the geometry optimizations, the QM subsystem was kept frozen and a 2 ns MD simulation was performed to allow the surrounding environment to reorient. The positions of the atoms in the active site were constrained, because the hydrogen bonding network (figure 1 in the paper) becomes unstable after the transfer. After the simulation, the complete QM/MM system was again optimized using the conjugate gradient method. Then, two QM/MM single point calculations were performed on each of the four systems to obtain the total energy of proton transfer, including re-organization. The QM subsystem in the first of these computations was described at the PM3 level and at RHF/6-31G* level in the second. The results are listed in table S1. For comparison these computations were repeated on the active site in vacuo. 


\begin{tabular}{|c|c|c|}
\hline pG & Vacuo & Protein + solvent \\
\hline PM3 & +61.896 & +87.62 \\
\hline RHF/6-31G* & +40.38 & +109.91 \\
\hline \multicolumn{3}{|c|}{} \\
\hline pR & Vacuo & Protein + solvent \\
\hline PM3 & +58.22 & -61.68 \\
\hline RHF/6-31G* & +44.39 & -52.28 \\
\hline
\end{tabular}

Table S1. energy differences before and after proton transfer in vacuo, and in the hydrated protein. These are total QM/MM energies reported in $\mathrm{kJ} / \mathrm{mol}$. The chromophore and the sidechains of Glu46 and Tyr42 were described at either the PM3 level or the RHF/6-31G* level of theory. The rest of the system was modelled by the GROMOS 96 forcefield.

Even when the re-orientation of the protein environment is taken into account, the energy difference of the fully hydrated protein system after and before proton transfer from the Glu46 sidechain to the chromophore is negative only if the chromophore is isomerized (table 1, right column). The protein environment makes the transfer energetically favourable. In vacuo, the transfer energy is positive both before and after the isomerization (table 1, middle column). Although the energy differences indicate that proton transfer is energetically favourable. Yoda $e t a^{l}$. recently carried out a similar calculations and also found that proton transfer was only possible after isomerization. However, they based their conclusion on in vacuo calculations of the x-ray model of PYP. Niether solvent nor counter ions were included which must casts doubt on whether their results are in fact truly meaningful.

\section{Free energy of proton transfer}

In addition to the computations discussed above, the difference in Gibbs free energy associated with the proton transfer was also estimated using classical mechanics. The thermodynamic integration formula ${ }^{2}$ can be used to predict the free energy difference (_G) between two states $(A$ and $B$ ) of a system:

$$
\Delta G_{A B}=\int_{\lambda_{A}}^{\lambda_{B}} d \lambda\left\langle\frac{\partial H(\lambda)}{\partial \lambda}\right\rangle_{\lambda}
$$

where $H$ is the total classical Hamiltonian of the system and _ the coupling parameter. The coupling parameter defines the path along which the system is transformed from state $A$ to state $B$. As the free energy is a state function the difference in free energy is independent of the path taken which also does not need to be physically meaningful. In our approach, the path was defined as follows. At lambda 0.0 (state $A$ ) the glutamic acid side chain is protonated and the chromophore is deprotonated (as in figure 1). At lambda 1.0 (state $B$ ) the chromphore is protonated and the glutamic acid is deprotonated. To go from state A to state B, the proton disappears on the glutamic acid and appears on the chromphore. This is realized by slowly evolving the proton of Glu46 to a so-called dummy atom, which has no non-bonded interactions with the rest of the system. At the same time a dummy on the chromophore is changed into a proton. The path of this transformation can be simply written as: $\left(1-\_\right)(\mathrm{GluH}+p \mathrm{caDUM})+(\mathrm{GluDUM}+p \mathrm{caH})$, where DUM and $p$ ca stand for the dummy atom and the chromophore ( $p$ coumaric acid) respectively. The mass of the dummy atom was the same as the mass of the proton.

In total $21 \mathrm{MM}$ simulations of $300 \mathrm{ps}$ were performed, each at a different value of lambda. All simulations were performed with the gromacs MD simulation package in conjunction with the GROMOS96 forcefield. The forcefield for the chromophore and simulation details are described elsewhere ${ }^{3}$. Non bonded interactions between the initial and final state were interpolated using a soft-core potential ${ }^{4}$, with the soft-core parameter _ set to 1.51 . The average $\overline{\partial \lambda} / \lambda$ was accumulated over the last 200 ps period of the simulations. The first 100 ps were considered necessary to equilibrate the system prior to data collection. With the 21 averages, the free energy was estimated by integrating equation (1) numerically using the trapezoidal method. The errors in the averages were calculated using a block averaging procedure ${ }^{5,6}$.

For comparison the procedure outlined above was not only performed using a protein configuration in which the chromophore had isomerized ( $\mathrm{pR}$, figure $1 \mathrm{~b}$ ), but also with configuration in which the chromophore had not isomerized (pG, figure 1a). We find that before the isomerization, the free energy is positive: $48.21 \pm 19.2 \mathrm{~kJ} / \mathrm{mol}$. After the isomerization, the energy is negative: $-111.9 \pm 13.8$. These results also support the conclusions in the paper that after the isomerization, the proton transfer is thermodynamically favorable.

\section{References}

(1) Yoda, M., Inoue, Y., Sakurai, M. J. Phys. Chem. B 2003, 107, 14569 -14575.

(2) Mark, A.E. In: Encyclopedia of computational Chemistry, von Rague Schleyer, P., ed., John Wiley \& Sons, 1998, 1070-1083.

(3) Groenhof, G., Lensink, M.F., Berendsen, H.J.C., Mark, A.E. Proteins 2002, 48, 212-219.

(4) Beutler, T.C., Mark, A.E., van Schaik, R.C., Geber, P.R., van Gunsteren, W.F. Chem. Phys. Lett. 1994, 222, 529-539.

(5) Allen, M.P.m Tildesley, D.J., Computer Simulation of Liquids. Oxford Science Publication, Oxford.

(6) Hess, B., J. Chem. Phys. 2002, 116, 209-217 


\section{Animations}

Two movies in MPEG1 format are included. Movie_I.mpeg shows a successful photo isomerization. It consists of 6 parts: a) a zoom on the active site, b) $200 \mathrm{fs}$ of ground state simulation, c) a photon absorption (white flash and color change of the chromophore atoms), d) evolution in the excited state, e) the radiationless transition back to the ground state (at $\mathrm{t}=372 \mathrm{fs}$, indicated by a color change), f) further evolution on the ground state. Movie_II.mpeg shows an unsuccessful photo isomerization. It consists of 5 parts: a) $50 \mathrm{fs}$ of ground state simulation, b) a photon absorption (white flash and color change of the chromophore atoms), c) evolution in the excited state, $d$ ) the radiationless transition back to the ground state (at $t=372$ fs, indicated by a color change), e) further evolution in the ground state. 